\title{
Immune Modulation Therapy and Imaging: Workshop Report
}

Anthony F. Shields ${ }^{1,2}$, Paula M. Jacobs², Mario Sznol ${ }^{3}$, Michael M. Graham ${ }^{4}$, Ron N. Germain ${ }^{2}$, Lawrence G. Lum ${ }^{5}$, Elizabeth M. Jaffee ${ }^{6}$, Elisabeth G.E. de Vries ${ }^{7}$, Sridhar Nimmagadda ${ }^{6}$, Annick D. Van den Abbeele ${ }^{8}$, David K. Leung ${ }^{9}$, Anna M. Wu ${ }^{10}$, Elad Sharon ${ }^{2}$, and Lalitha K. Shankar ${ }^{2}$

${ }^{1}$ Karmanos Cancer Institute, Wayne State University, Detroit, Michigan; ${ }^{2}$ National Cancer Institute, Bethesda, Maryland; ${ }^{3}$ Yale University, New Haven, Connecticut; ${ }^{4}$ University of Iowa, Iowa City, Iowa; ${ }^{5}$ University of Virginia, Charlottesville, Virginia; ${ }^{6}$ Johns Hopkins University, Baltimore, Maryland; ${ }^{7}$ University of Groningen Medical Center, Groningen, The Netherlands; ${ }^{8}$ Dana-Farber Cancer Institute and Brigham and Women's Hospital, Harvard Medical School, Boston, Massachusetts; ${ }^{9}$ Bristol-Myers Squibb, Princeton, New Jersey; and ${ }^{10}$ Crump Institute for Molecular Imaging, David Geffen School of Medicine at UCLA, Los Angeles, California

A workshop at the National Cancer Institute on May 2, 2016, considered the current state of imaging in assessment of immunotherapy. Immunotherapy has shown some remarkable and prolonged responses in the treatment of tumors. However, responses are variable and frequently delayed, complicating the evaluation of new immunotherapy agents and customizing treatment for individual patients. Early anatomic imaging may show that a tumor has increased in size, but this could represent pseudoprogression. On the basis of imaging, clinicians must decide if they should stop, pause, or continue treatment. Other imaging technologies and approaches are being developed to improve the measurement of response in patients receiving immunotherapy. Imaging methods that are being evaluated include radiomic methods using CT, MRI, and ${ }^{18} \mathrm{~F}-\mathrm{FDG}$ PET, as well as new radiolabeled small molecules, antibodies, and antibody fragments to image the tumor microenvironment, immune status, and changes over the course of therapy. Current studies of immunotherapy can take advantage of these available imaging options to explore and validate their use. Collection of CT, PET, and MR images along with outcomes from trials is critical to develop improved methods of assessment.

Key Words: immunotherapy; biomarkers; imaging cancer

J Nucl Med 2018; 59:410-417

DOI: 10.2967/jnumed.117.195610

\section{$\mathbf{T}$} he Clinical Trials Network of the Society of Nuclear Imaging and Molecular Therapy (SNMMI) cosponsored a workshop with the National Cancer Institute (NCI) on Monday, May 2, 2016, in Rockville, MD, titled, "Immune Modulation Therapy and Imaging: What Can We Do in Clinical Trials Now?" A video archive of the meeting, transcripts, and slides are available through the NCI and SNMMI $(1,2)$. This imaging workshop was inspired by the rapid growth in the study and use of immunotherapy, which has shown some remarkable responses in the treatment of tumors. However, these responses are quite variable, and early anatomic imaging may show that a tumor has increased in size.

Received May 2, 2017; revision accepted Jul. 11, 2017.

For correspondence or reprints contact: Anthony F. Shields, Karmanos Cancer Institute, 4100 John R. St., HW04HO, Detroit, MI 48201.

E-mail: shieldsa@karmanos.org

Published online Aug. 17, 2017.

COPYRIGHT (C 2018 by the Society of Nuclear Medicine and Molecular Imaging.
Although overt pseudoprogression is uncommon, minor increases in tumor size and delayed responses are often seen. On the basis of imaging, clinicians must decide if they should stop, pause, or continue treatment, and this has led to the development of immune response criteria, which remain imperfect. This workshop considered other technologies and imaging approaches to improve response evaluation in patients receiving immunotherapy agents that stimulate the immune response, including antibodies against the checkpoint inhibitors cytotoxic T-lymphocyte-associated antigen 4 (CTLA-4), programmed death 1 (PD-1), and programmed death ligand 1 (PD-L1). Initial ipilimumab monotherapy in patients with melanoma can result in pseudoprogression and delayed response, with overall benefit in only about $20 \%$ of patients (3). Combinations of checkpoint inhibitors, in melanoma, have resulted in response rates of over $50 \%$ but elicit considerable toxicity (4).

Some tumor biomarkers, such as T-cell infiltration and PD-L1 expression, identified using biopsies from patients undergoing immunotherapy have been helpful in predicting the response before treatment. Imaging may add specificity and improve assessment. This workshop concentrated on technologies that can be applied in the clinic now and in the near future. New uses of available PET tracers, such as ${ }^{18} \mathrm{~F}-\mathrm{FDG}$ and ${ }^{18} \mathrm{~F}$-fluorothymidine, as well as several new tracers and image analyses have the potential to be incorporated into clinical trials either ongoing or just getting under way.

\section{IMMUNOLOGY OVERVIEW AND CURRENT ROLE OF IMAGING}

Simplistically, producing immune-mediated tumor regression depends on modulating a preexisting tumor antigen-specific T-cell response or inducing such a response. As reported at the meeting by Dr. Sznol, at least a subset of patients with advanced malignancies has an inflamed tumor microenvironment (TME), and these tumors may contain $\mathrm{T}$ cells bearing receptors that recognize tumor antigens (5). In metastatic melanoma, for example, tumorinfiltrating lymphocytes can be isolated from most patients, and some of the CD8-positive lymphocytes within the tumor express PD-1 (6), identifying the subset that demonstrates tumor recognition in vitro. Cytokine-induced upregulation of the PD-1 ligand PD-L1 within nearby tumor cells or other immune cells constrains subsequent T-cell antitumor function (7). Remarkably, antibody blockade of PD-1 or PD-L1 produces clinically meaningful tumor responses in a subset of patients across a broad range of solid tumors and hematologic malignancies (8). 
Current clinical development is focused on blocking other immune regulatory checkpoints, providing immune costimulatory signals, inducing immune responses with vaccines, or increasing traffic of lymphocytes into the tumor. Hundreds of drug combinations are currently in development. Major challenges include the lack of accurate predictive biomarkers and poor understanding of the critical and nonredundant signals necessary to produce immune-mediated tumor regression in individual patients.

Immune therapy development could be facilitated by novel imaging modalities as improved predictors of which patients could benefit from PD-1 or PD-L1 blockade alone or from combinations.

\section{T-CELL THERAPIES}

Current imaging of solid tumors during and after immunotherapy cannot distinguish inflammatory immune responses from infiltration or infused effector cells, endogenous immune cells recruited to the site, tumor progression, or a combination. Dr. Lum described clinical studies using anti-CD3 $\times$ anti-human epidermal growth factor receptor 2 (HER2) bispecific antibody-armed activated $\mathrm{T}$ cells, in which several strategies were used to evaluate trafficking, infiltration of tumors, in vivo survival, and the induction of endogenous immunity (9). In a phase I clinical trial of 23 heavily pretreated women with metastatic breast cancer, armed $\mathrm{T}$ cells were detected by staining tumor biopsies. Using conventional ${ }^{18}$ F-FDG PET/CT to assess clinical responses, nearly complete clearance of multiple liver and bony metastases was seen in 1 patient (10). However, the striking observation was that the median overall survival was 57 mo for the HER2-positive group with $3+$ staining $(n=8)$ and 28 mo for the HER2-negative group $(n=15)$. Many of the patients who were stable at 14.5 wk after initiation of therapy later progressed and received the oncologist's choice of chemotherapy. These results suggested that patients may have had ${ }^{18} \mathrm{~F}-\mathrm{FDG}-$ positive flare responses before receiving a subsequent line of chemotherapy that stabilized them. Recent results from checkpoint inhibitor trials using immune RECIST indicate that immune responses are delayed and may involve a tumor flare that confounds interpretation of scans (11). To minimize tumor growth effects on ${ }^{18}$ F-FDG PET/CT scans, in one selected patient Lum's group assessed trafficking to tumors or the inflammatory immune response by scanning within days after chemotherapy and rescanning immediately after 2 infusions of armed T cells (12). The scans clearly and strongly suggested trafficking or an inflammatory reaction at tumor sites within 3-5 d after the infusions (Fig. 1). A second patient with unresectable pancreatic cancer who was stable on chemotherapy received anti-CD3 $\times$ anti-epidermal growth factor receptor bispecific antibody-armed T cells (12). Tumor flare suggested rapid progression, but the patient developed a complete response shortly after restarting chemotherapy. The latter case illustrates the need to develop approaches that can distinguish between immune responses and tumor progression.

Preclinical studies with new imaging agents that label monoclonal antibodies and purified T-cell subsets for trafficking and imaging are encouraging. With new agents that mark immune cells, targeted $\mathrm{T}$ cells, or T-cell subsets, gene-transduced chimeric antigen receptor $\mathrm{T}$ cells will provide clinically and biologically important data in adoptive clinical trials. In new strategies involving dosing of $\mathrm{T}$ cells, there are several variables in obtaining clinically and biologically meaningful data, including the type of imaging agent or label, the timing of the imaging studies, the types of tumors, and the tumor sites to be imaged. Development of a consistent and reliable imaging system that provides the clinician with scans that differentiate tumor flare from tumor progression would be helpful in guiding decision making.

\section{VACCINE THERAPY}

The number of patients who benefit from checkpoint inhibitors for specific tumor types is not inconsequential: although only about $20 \%-40 \%$ benefit as demonstrated by tumor shrinkage, others may benefit without clear decreases in tumor size. Dr. Jaffee described the need to better understand how to increase the percentage of patients who respond. For example, pancreatic cancer is particularly immunoresistant, having a significant desmoplastic stromal reaction that is immunosuppressive and contains very few regulatory T cells. Mouse models of pancreatic cancer show acute and then chronic inflammation, leading to the stromal reaction and to studies using whole-tumor vaccines to boost fully mature dendritic cells in the tumor. When the vaccines are given to patients before surgery, the tumors develop more lymphoid aggregates. New approaches are needed to assess such responses.

Using a vaccine may alter the expression of PD-L1 and PD-1 in tumors and immune cells (13), encouraging studies comparing neoadjuvant vaccines alone or in combination with anti-PD-1 antibodies. Combinations are also being explored in patients with metastatic disease. Early trials have shown activity in patients treated by vaccination along with ipilimumab (14). The response, however, can be delayed for weeks or months. Many different combinations are possible, but there is presently no way to assess whether the patient is truly responding. We need to understand the TME of the patients and whether it is being changed by a boost from a vaccine. We could personalize treatment if we could image the TME in real time and evaluate other modulating agents that may be given at the same time.

\section{CLARIFYING RESPONSE CRITERIA}

The goal of cancer therapy is to improve patient survival. Therefore, as presented by Dr. Leung at the meeting, discussions of tumor response assessment must be in the context of survival benefit rather than empiric metrics such as tumor shrinkage. Although chemotherapy and some targeted therapies have demonstrated improvement in short-term survival, the long-term prognoses for some of the new therapies are barely better than for controls (15).

Evidence suggests that response durability improves with immunotherapy (15), which uniquely modulates the body's ability to attack tumor cells (16). These changes in the immune system are likely the basis for long-term benefits such as sustained immunity against infections. In a subgroup analysis of a randomized phase 2 trial of metastatic renal cell cancer, $69 \%$ of the patients who continued immunotherapy beyond RECIST-defined progression subsequently experienced tumor reduction or stabilization, as well as showing a longer overall survival than those who discontinued therapy (median, 22.5 vs. $12.3 \mathrm{mo}$ ) (17). This analysis could be biased since those with minor progression or who appear clinically stable may do better than those with more obvious clinical deterioration.

Consequently, in developing the next-generation response criteria, we need to ensure that the revised standard accommodates trial designs independent of therapy type. One step is to expand imaging parameters beyond conventional unidimensional (e.g., RECIST) or bidimensional (e.g., World Health Organization, 


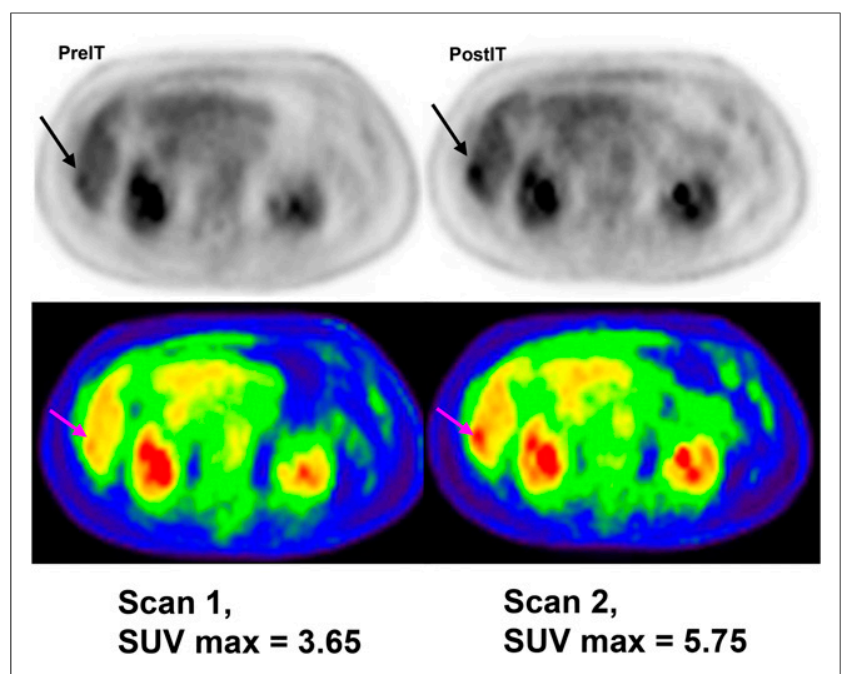

FIGURE 1. ${ }^{18} \mathrm{~F}$-FDG PET before and 2 wk after starting treatment of HER2 bispecific antibody-armed activated T cells shows inflammatory response.

Response Assessment in Neuro-Oncology Criteria) size measurements $(11,18)$. Mozley et al. showed that tumor volume measurements were highly sensitive in detecting tumor response and progression (19). ${ }^{18} \mathrm{~F}$-FDG PET/CT is now a standard imaging tool in determining response to therapy in lymphomas. Dynamic models of tumor growth have been used to derive tumor-response metrics shown to be predictive of survival (20).

Additionally, the development of new response criteria must use all other data to maximize our ability to reflect tumor and immune biology and interactions. Incorporation of nonimaging metrics into a multimodality efficacy metric will contribute to better predicting tumor response, identifying resistance mechanisms, rationally selecting immune and other therapeutics, and ultimately improving patients' lives.

\section{IMAGING CAPABILITIES}

Nuclear medicine offers several radionuclides to label a range of tracers of interest. For example, as discussed by Dr. Graham, SPECT provides the capability of imaging the distribution of ${ }^{99 \mathrm{~m}} \mathrm{Tc}-,{ }^{111} \mathrm{In}-,{ }^{123} \mathrm{I}-,{ }^{131} \mathrm{I}-$, and ${ }^{201} \mathrm{Tl}$-labeled compounds. Although ${ }^{18} \mathrm{~F}$ is the most commonly used radionuclide with PET, an expanding repertoire of tracers is being labeled with ${ }^{11} \mathrm{C}$, ${ }^{68} \mathrm{Ga},{ }^{64} \mathrm{Cu}$, and ${ }^{89} \mathrm{Zr}$.

Metabolic imaging, receptor imaging, and cell trafficking are likely to be the most useful ways to monitor response to immune modulation therapy, and many new approaches are moving through in vitro, cell culture, and animal studies before testing in humans (21). Receptor imaging is powerful and likely to succeed because there are numerous cell-surface receptors involved in cell-signaling pathways. Labeled receptor ligands that have real potential to be useful in characterizing immune modulation therapy include a CD-20 agent, approved for treating lymphoma (rituximab), and pentixafor, which targets $\mathrm{CXC}$ chemokine receptor 4 , a receptor upregulated in myeloma. Work on labeling anti-PD-1 or anti-PD-L1 has just begun.

Cell-trafficking labeling has not yet been explored sufficiently. Since the goal in many immune modulation therapies is activation of lymphocytes, tracking them may be predictive of response to therapy. The widely used approaches for labeling cells with ${ }^{111}$ Inoxine or ${ }^{99 \mathrm{~m}} \mathrm{Tc}-\mathrm{HMPAO}$ could be a powerful way to help understand what is happening in the individual patient.

\section{BIOMARKERS OF IMMUNE MODULATION}

The immune system comprises many distinct cell types that contribute to adaptive and innate immunity, alongside a plethora of soluble mediators that have direct effector function as well as contributing to cell migration and localization. The recent success of immunotherapies involving engineered $\mathrm{T}$ cells (chimeric antigen receptor T cells) (22) and drugs that interfere with checkpoints limiting adaptive cell-mediated immunity, including those directed to CTLA-4 and PD-1 or PD-L1 (23), have emphasized the importance of immune responses in successful treatment of a variety of cancers. A robust immune response is also needed for successful chemotherapy (24).

These emerging findings underline the need to improve metrics for how a given patient's system is responding to a cancer, as discussed by Dr. Germain. Patients lacking a measurable cellmediated response to their tumor may not respond to checkpoint blockade or vaccination but may be successfully treated with chimeric antigen receptor $\mathrm{T}$ cells, whereas those with a weak but measurable response may respond well to a combination of carefully timed chemotherapy or radiation together with checkpoint blockade and vaccination to boost adaptive immunity. One area requiring further development—and promising especially useful and direct information about the interaction of tumors with the immune system-is fine-grained analysis of the TME itself at the microscopic level. New methods are emerging for highly multiplexed analysis of cell types, signaling, and functional state, linked to correlation of these data types with response outcome, potentially leading to new ways of using pathologic examination of tumor samples in guiding treatment decisions.

Histocytometry is a recently developed method that allows multiplexed (14 or more parameters at a time, possibly 30-40 with limited reprobing of a sample), high-resolution imaging of tumor samples (Fig. 2) (25). By the right choice of reagents, cells can be phenotyped at a level approaching modern flow cytometry while preserving spatial information of importance in understanding immune cell-tumor cell interactions in the TME. Combining these powerful imaging tools with data from other technologies that rely on peripheral blood sampling will permit the rapid development of new assays to classify cancer patients for appropriate therapy and to learn why some treatments do not work in certain individuals, aiding in the development of future novel interventional strategies.

\section{IMAGING INFLAMMATION WITH ${ }^{18}$ F-FDG, ${ }^{18}$ F-FLT, AND BEYOND}

With immunotherapy, knowledge of the TME is essential in evaluating the effectiveness of an immune response that may evolve at the tumor site or at other potential therapeutic targets involved in the cancer-immunity cycle, such as lymph nodes and spleen (26). More than one biomarker will be required for qualitative and quantitative determination of antitumor immunocompetency and therapeutic response.

${ }^{18}$ F-FDG PET/CT, the most commonly used functional imaging test in patients with cancer, is not specific to cancer because high 


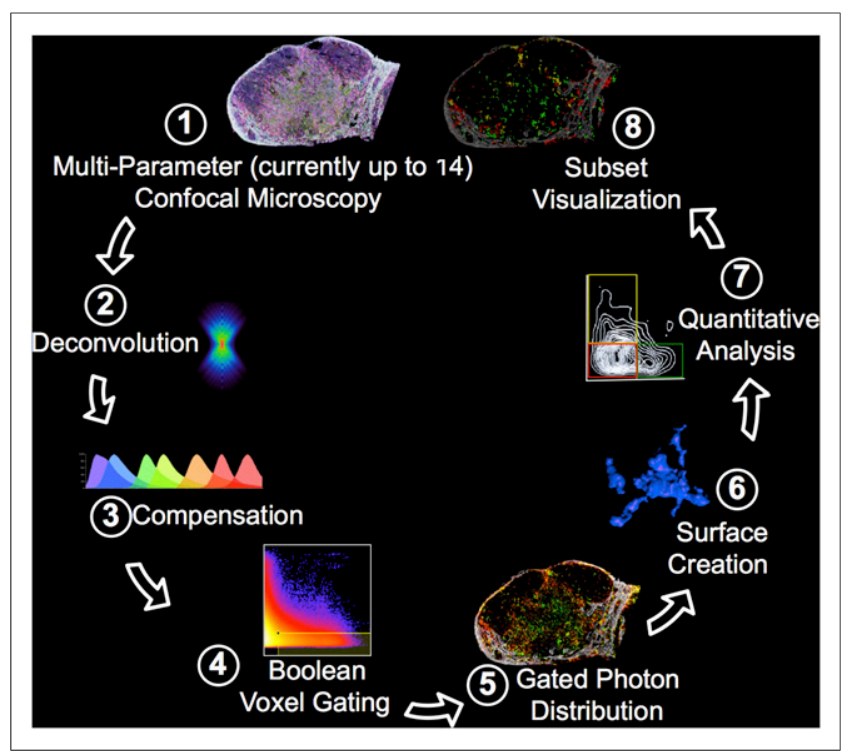

FIGURE 2. Histocytometry processing and analysis of tissue sections. (1) Fixed tissue section is simultaneously stained with antibodies directed to as many as 14 determinants and imaged in tiled, high-resolution mode using advanced confocal microscope. (2) Image data are deconvoluted to improve signal-to-noise ratio and spatial resolution. (3) Spillover between fluorescent emissions is corrected (compensated). (4-6) The resulting data are used to create cellular objects based on membrane staining, with defined objects retaining all fluorescence information associated with that object. (7) These data are equivalent to flow cytometry data and can be processed using software for flow data analysis, yielding quantitative information about cellular subset frequency (for example) or intensity of staining for a given determinant. (8) Because data also retain spatial $x-y-z$ information about each object, each gated and defined cellular object can also be placed in its original tissue location.

glucose metabolism can also be seen in inflammatory cells. As noted by Dr. Van den Abbeele, detecting an inflammatory reaction may be confounding when assessing response at the tumor site but can be useful to diagnose immune-related adverse events early in patients treated with immunotherapy, sometimes weeks before the development of symptoms, allowing timely initiation and assessment of response to corticosteroid therapy (27).

In the imaging assessment of tumor response to immunotherapy, both standard anatomic measurements of tumor size on CT or MRI (such as RECIST 1.1) (28) and measurement of metabolic response by ${ }^{18} \mathrm{~F}$-FDG PET (European Organization for Research and Treatment of Cancer criteria) (29) can be combined when there is no significant decrease in tumor size but also no evidence of new tumors during or after completion of treatment.

When compared with cytotoxic chemotherapy or molecularly targeted therapy, cancer vaccines and immunomodulatory monoclonal antibodies often show unconventional patterns of tumor response, including delayed response, clinically significant disease stability, transient enlargement of tumor size, and the appearance of new tumors for weeks and months over the course of therapy (27). Several immune-related response criteria have been proposed using bidimensional measures (such as immune-related response criteria) or unidimensional measures (such as immune RECIST) $(11,18,28)$. The drawback of all anatomically based criteria is that they still rely on a size measurement that may or may not reflect therapeutic response and still require imaging to be repeated weeks later before being able to confirm clinical benefit (or lack thereof).

Molecular imaging, on the other hand, can dissect all the hallmarks of cancer biology. For example, after PD-1 blockade, proliferating CD8-positive T cells localize to the tumor and directly correlate with eventual radiographic reduction in tumor size (30). Noninvasive imaging with ${ }^{18} \mathrm{~F}$-fluorothymidine characterized the response in patients with advanced melanoma receiving tremelimumab, an anti-CTLA-4 immune checkpoint inhibitor (31).

More sensitive and specific biomarkers for inflammatory cells and the TME are needed and are being actively tested in preclinical and clinical settings (Supplemental Table 1; supplemental materials are available at http://jnm.snmjournals.org) (32).

Also, with advances in analytic tools, machine learning, data storage, and big data analysis, the field of radiomics or radiogenomics is learning to decode the tumor phenotype by extracting and analyzing large amounts of advanced quantitative imaging features from standard-of-care clinical CT, PET, or MRI scans (33). Imaging is well placed to integrate with panomics, molecular pathology, clinical data, and outcome analyses to better characterize the tumor; enrich trials with improved patient selection, clinical trial design, and biologically relevant drug dosing; and enable faster translation of discovery and drug development. Although challenges remain and validation of these computer-aided techniques has just begun, integrating radiomics and radiogenomics tools with our clinical and research efforts may have a significant impact on clinical and research decision making in the future by providing unique information at the point of care.

\section{ANTIBODY IMMUNOTHERAPY IMAGING}

Immunotherapy with immune checkpoint-inhibiting antibodies is a novel, rapidly evolving field. Patient selection based on factors such as PD-L1 expression and somatic mutations in tumor biopsies underestimates the complexity and dynamics of immune response.

Dr. de Vries discussed modern PET technology and tracers that enable noninvasive whole-body imaging to determine whether the antibody reaches the target; the group also provided quantitative measurements of target expression and potential heterogeneity in tracer tumor uptake (34). The antibodies that act as immune checkpoint inhibitors have activity across tumor types, but not all patients respond and major side effects can occur. PET may potentially allow personalized immunotherapy decisions.

Imaging and therapy with radiolabeled antibodies have been studied for many years and continue to be widely examined $(35,36)$. One problem has been the heterogeneity of uptake. For example, in one study, in approximately $30 \%$ of the patients with HER2positive tumors, ${ }^{89} \mathrm{Zr}$-trastuzumab was taken up by the tumor lesions and was heterogeneous (37). Current preclinical imaging has demonstrated that radioactive and fluorescence-labeled PD-L1 antibodies show specific tumor uptake and are internalized in tumor cells but also show high uptake in the spleen, lymph nodes, and thymus $(38,39)$.

There are ongoing clinical trials with ${ }^{89} \mathrm{Zr}$-labeled immune checkpoint inhibitors. In one trial, ${ }^{89} \mathrm{Zr}$-atezolizumab PET is being performed before treatment with the PD-L1 antibody atezolizumab to study tracer uptake in primary and metastatic tumors and normal tissues and to evaluate the future value of ${ }^{89} \mathrm{Zr}$-atezolizumab PET for patient selection (ClinicalTrials.gov identifier NCT02453984). 
In another trial, the in vivo whole-body distribution of the anti-PD-1 PET tracer ${ }^{89} \mathrm{Zr}$-pembrolizumab is being evaluated in locally advanced metastatic melanoma or non-small cell lung cancer lesions before pembrolizumab treatment (ClinicalTrials.gov identifier NCT02760225). Additional studies are ongoing with smaller labeled bispecific antibody (construct) tracers. Preclinically, specific tumor uptake has been shown by an ${ }^{89} \mathrm{Zr}$-labeled bispecific T-cell-engaging antibody construct targeting epithelial cell adhesion molecule or carcinoembryonic antigen (40), and clinically, an ${ }^{89} \mathrm{Zr}$-labeled bispecific T-cell-engaging carcinoembryonic antigen antibody is being studied (ClinicalTrials.gov identifier NCT02291614). Besides imaging with radioactive labeled antibodies, imaging is also possible with fluorescently labeled antibodies and optical imaging as shown in patients with breast cancer (41).

\section{LABELED ANTIBODY FRAGMENTS FOR IMAGING}

Labeled antibodies for PET imaging provide the sensitivity, resolution, and quantification for monitoring immune cells and their roles in immune responses. Thus far, a variety of approaches has been developed for detection of immune cells in vivo (Supplemental Table 2) (42). Metabolic probes such as ${ }^{18} \mathrm{~F}-\mathrm{FDG}$ and ${ }^{18} \mathrm{~F}$-fluorothymidine assess elevated glycolysis and DNA synthesis, respectively, and have broad clinical applications. However, elevated ${ }^{18} \mathrm{~F}$-FDG or ${ }^{18} \mathrm{~F}$-fluorothymidine uptake is relatively nonspecific, being common in both tumor cells and activated immune cells, thus confounding interpretation. Ex vivo cell labeling with ${ }^{111}$ In-oxine is well established for imaging infection. Methods under development include ${ }^{89} \mathrm{Zr}$-oxine for PET and a variety of nanoparticles (iron oxide; ${ }^{19} \mathrm{~F}$ ) as contrast agents for MRI. However, these approaches require removal, manipulation, and reinfusion of cells into patients and can be limited by radioactive decay or dilution of signal as cells divide in vivo. Reporter genes can provide long-term monitoring of immune cells as they home and expand. Central to their clinical development is the requirement for nonimmunogenic reporter genes; furthermore, this approach is restricted to applications in which cells can be genetically modified in situ or ex vivo. Given these challenges, direct imaging of endogenous cell-surface targets, using antibodies, fragments, or scaffolds such as nanobodies, is promising, as reviewed by Dr. Wu.

Engineering of antibodies enables optimization of characteristics critical for their use as clinical imaging agents (43). Engineered antibody fragments, such as minibodies ( $\mathrm{scFv}$ [single-chain variable-fragment]- $\mathrm{C}_{\mathrm{H}} 3$ dimers) and diabodies ( $\mathrm{scFv}$ dimers), incorporate features specifically optimized for imaging, including retention of the specificity and affinity of the parental antibody, bivalency, and accelerated clearance (for same-day or next-day imaging). Additionally, the availability of nonstandard positron-emitting radionuclides has accelerated the development of PET tracers based on biologicals. Positron-emitting radiometals such as ${ }^{64} \mathrm{Cu}$ (half-life, $12.7 \mathrm{~h}),{ }^{89} \mathrm{Zr}$ (3.2 d), and ${ }^{124} \mathrm{I}$ (4.2 d) allow a closer match between physical and biological characteristics.

Preclinical studies of engineered antibody fragments such as cys-diabodies have shown utility for detection of CD8 and CD4 T lymphocytes in mouse models of hematopoietic stem cell reconstitution (44) and tumor immunotherapy (45). An ${ }^{89} \mathrm{Zr}$-desferrioxamine anti-CD8 cys-diabody detected tumor infiltration of cytotoxic CD8 $\mathrm{T}$ cells in mice treated with a checkpoint inhibitor; patterns and levels of uptake differed between responders and nonresponders (Fig. 3 , left). Antigen-driven homing and expansion of adoptively transferred ovalbumin-specific T cells were visualized by CD8 PET (Fig. 3, right).

PET imaging with humanized or human antibodies or fragments is amenable to clinical translation. For example, an ${ }^{89} \mathrm{Zr}-$ desferrioxamine anti-prostate-specific membrane antigen minibody demonstrated effective lesion targeting and visualization in men with metastatic prostate cancer (Fig. 4 (46)). Similar approaches using engineered antibodies targeting human CD8, CD4, and other immune cell-specific targets should prove useful for profiling immune responses in patients undergoing cancer immunotherapy. Developing such tracers will not be easy.

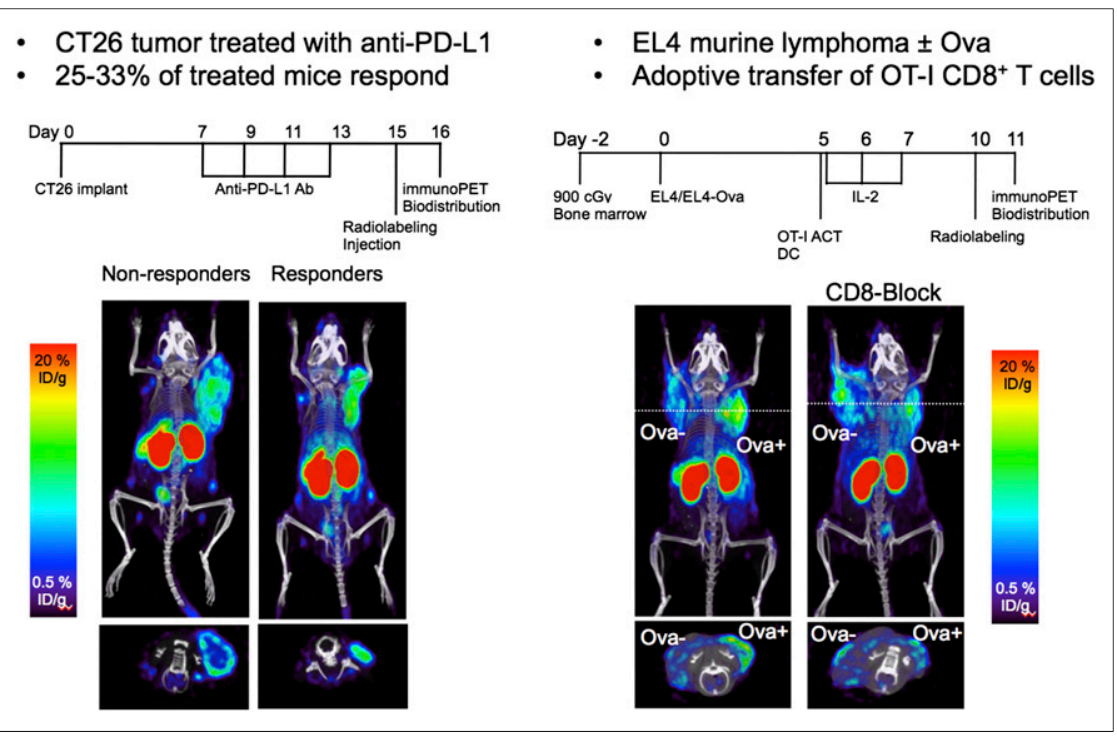

FIGURE 3. Imaging CD8 T-cell infiltration in tumor immunotherapy. Anti-CD8 PET was performed $5 \mathrm{~d}$ after adoptive transfer of OT-1 ovalbumin-specific T cells in C57BL/6 mice bearing subcutaneous Ova-negative and Ova-positive EL4 tumors. Mouse on right was preblocked with cold anti-CD8 antibody.

\section{IMAGING WITH CHEMOKINES AND SMALL MOLECULES}

T-cell activity at the tumor is a main driver of immune modulation therapy; imaging agents specifically focused on activated $\mathrm{T}$ cells, however, are few. The metabolic pathway and the cytokine/chemokine pathway are central in the immune TME and in defining tumor response to immune modulation therapies. To monitor activated T-cell activity at the tumor, Dr. Nimmagadda focused on these two important pathways as possible targets for imaging agents.

Metabolic reprogramming is required for $\mathrm{T}$ cells to exert effector function at the tumor. $\mathrm{T}$ cells in immune surveillance in an energy-oriented oxidative metabolic state must undergo a metabolic shift to a state that supports rapid growth to exert effector function. Also, cancer cell-induced nutrient deprivation affects T-cell signaling and gene expression within the TME. For 


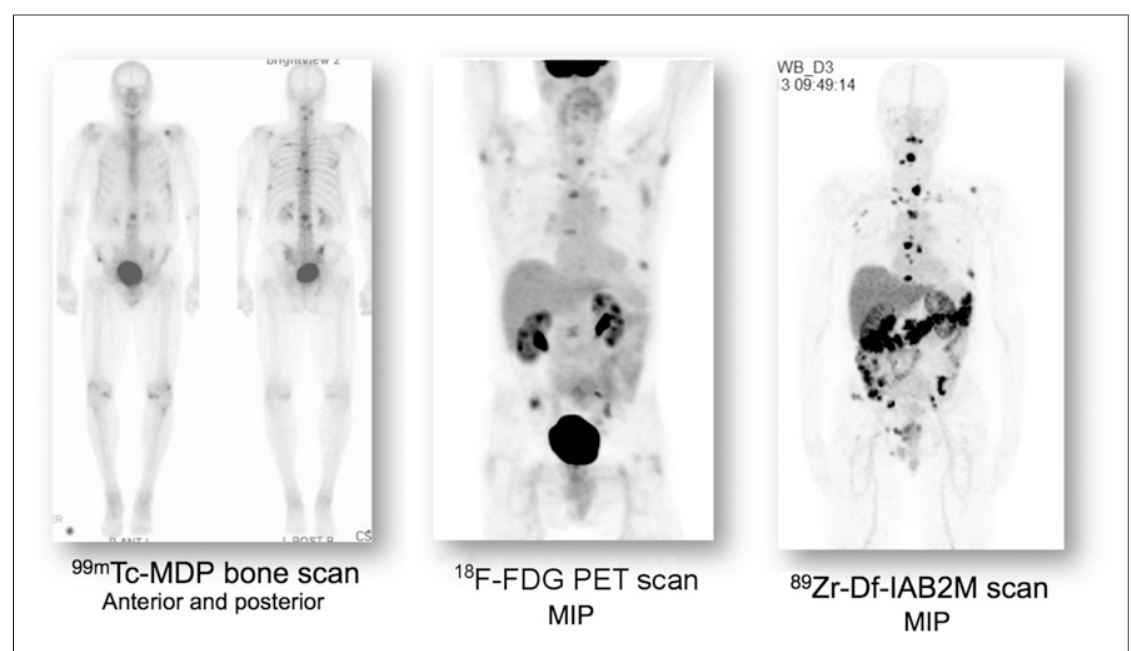

FIGURE 4. First-in-human imaging with ${ }^{89} \mathrm{Zr}$-desferrioxamine (Df)-IAb2M anti-prostate-specific membrane antigen minibody in patients with metastatic prostate cancer, compared with $99 \mathrm{mTc}$ methylene diphosphonate (MDP) bone scan and ${ }^{18}$ F-FDG PET maximum-intensity projection (MIP).

example, tryptophan depletion in the TME limits T-cell activation and effector function. Increased upregulation of two enzymes in tryptophan metabolism-indoleamine 2,3-dioxygenase and tryptophan 2,3 dioxygenase-by cancer cells and antigen-presenting cells increases metabolism of tryptophan (47), and high tumor indoleamine 2,3-dioxygenase levels correlate with poor prognosis. Indoleamine 2,3-dioxygenase inhibitors and tryptophan analogs are being investigated as therapeutics and imaging agents to target the immunosuppressive TME (48). Another agent that is being developed as a potential T-cell-imaging agent is the ${ }^{18} \mathrm{~F}$-labeled guanosine analog 2 '-deoxy-2'-fluoro-9- $\beta$-D-arabinofuranosylguanine (49), a specific substrate for mitochondrial deoxyguanosine kinase that is upregulated in activated T cells. Preliminary in vitro and in vivo studies have demonstrated uptake of this guanosine analog in activated T cells (49) and have led to a clinical trial (NCT02323893). Imaging of the immune checkpoint protein PD-L1 is being developed using antibodies, but small peptide- based PET agents may also prove useful and can be labeled with ${ }^{64} \mathrm{Cu}$ for imaging (Fig. 5) (50).

Chemokine gradients and chemokine receptor expression on immune cells play a critical role in immune cell migration and homing to the tumors, contributing to the modulation of the immune TME. One promising target for imaging-agent development is CXC chemokine receptor 3, because both preclinical and clinical studies (51) support it as an effector cell marker. Imaging agents have not yet been developed for this receptor, but many high-affinity small-molecule inhibitors have potential as imaging agents.

\section{BRIDGING THE GAPS TO INVESTIGATIONAL-NEW-DRUG AND NCI SUPPORT}

The NCI has resources that can assist with developing essential data for obtaining regulatory permission to test a drug in humans or for obtaining an investigational-new-drug exemption in the United States, as well as assisting with the equivalent requirements in other countries. As discussed by Dr. Jacobs, research grants are available in several areas: general funding (R01, R21, R03), specialized imaging-specific initiatives, small-business grants, the NCI Experimental Therapeutics Program, the NCI National Clinical Trials Network, and even some regulatory advice. Additionally, the Food and Drug Administration has a grant program for the development of orphan drugs (Supplemental Table 3).

The NCI Experimental Therapeutics Program provides direct access to NCI resources and expertise, but it is not a grant. The NCI performs approved portions of the project for the applicant using its internal resources. There is a simple application process with both internal and external review panels, and the applicant works with the NCI staff on the project. The NCI Experimental Therapeutics Program also provides regulatory and toxicology

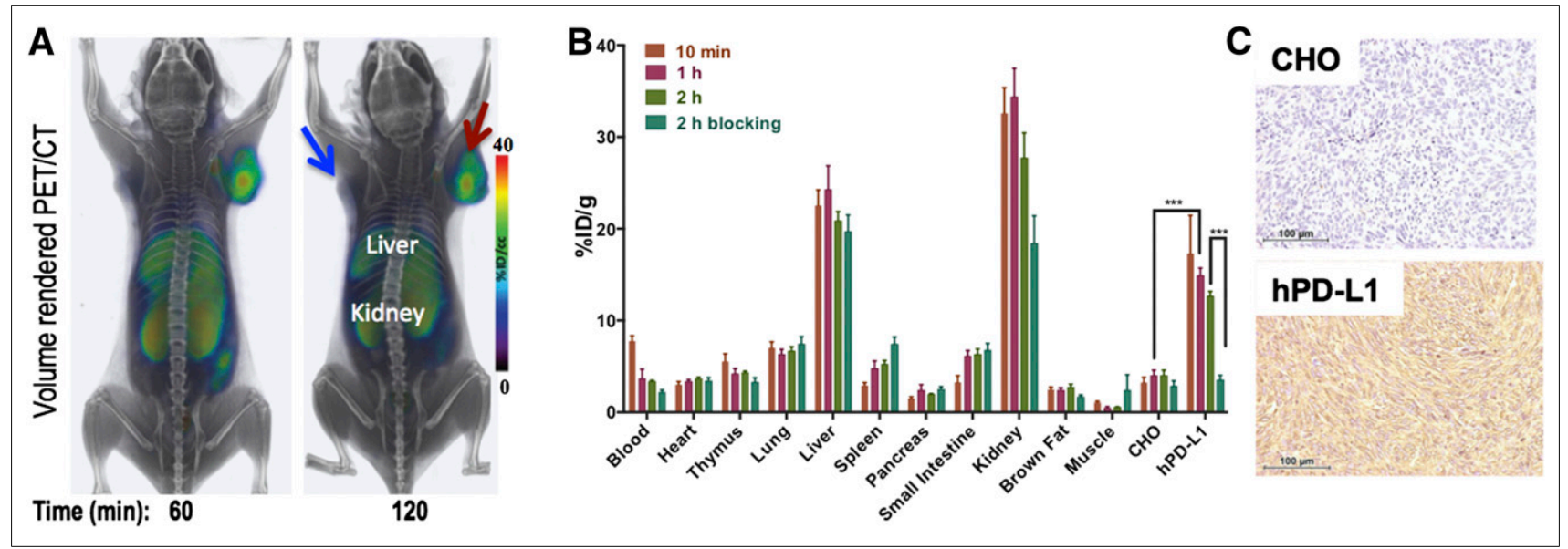

FIGURE 5. (A) Volume-rendered PET/CT images at 60 and $120 \mathrm{~min}$ after intravenous administration of $5,550 \mathrm{MBq}(150 \mathrm{mCi})$ of ${ }^{64} \mathrm{Cu}-\mathrm{WL} 12$ to NSG mice show specific accumulation in hPD-L1 tumor (red arrow) as opposed to CHO tumor (blue arrow). (B) Percentage injected dose per gram of tissue at 10, 30, 60, and 120 min after tracer injection. (C) Photomicrographs illustrating the typical histologic patterns of the two tumor types. 
advice in advance of an application (https://next.cancer.gov/ experimentalTherapeutics/form.htm).

Additionally, the NCI Cancer Imaging Program provides regulatory resources that are more imaging-focused. It has filed several INDs for investigational trials and will provide cross-file letters to independent principal investigators. Additionally, full standard operating procedures for manufacturing the tracers ${ }^{18} \mathrm{~F}$-fluorothymidine, $16-\alpha-{ }^{18} \mathrm{~F}$-fluoroestradiol, ${ }^{18} \mathrm{~F}$-FMISO $(1 \mathrm{H}-1-$ (3- ${ }^{18} \mathrm{~F}$-fluoro-2-hydroxy-propyl)-2-nitro-imidazole), and ${ }^{89} \mathrm{Zr}$ panitumumab are available to download.

The NCI National Clinical Trials Network conducts large-scale, multicenter trials of drugs and imaging agents after they have successfully completed early trials.

\section{NEXT STEPS}

The situation today is different from that 5 years ago because we have numerous new treatments, including an expanding array of immunotherapies. At the NCI, about $23 \%$ of intramural research projects involve immunotherapy, and an increasing number of extramural grants also involve immunotherapy. Between 2010 and 2015, the immunotherapy trials activated in the NCI National Clinical Trials Network numbered 88, and in 2014-2015 they made up $22 \%$ of the portfolio, including studies for both advanced disease and adjuvant therapy.

Given the cost and toxicity of these agents, as well as the variable response to them, we have a limited ability to predict and monitor the efficacy of such treatments. Performing national trials using genomic testing requires an extraordinary effort to validate tests across multiple testing centers, as in the Molecular Analysis for Therapeutic Choice (MATCH) trial. Imaging studies have also been problematic. Image reconstruction algorithms vary from vendor to vendor and among devices, whether CT, MRI, or PET. Commercial vendors and the imaging community need to work together to ensure that acquisition protocols and image analyses are standardized and reproducible. The field of radiomics may provide useful information on the TME and heterogeneity, but we must standardize the methods of acquisition and analysis to ensure reproducibility in longitudinal studies.

Central collection of images from many trials is ongoing. These image sets can help explore and validate the use of radiomics. To determine whether there are image signatures that predict the aggressiveness of tumors, the response to therapy, and early markers of outcome, we need to know which imaging approaches are being used in ongoing trials. Clinicaltrials.gov identifies therapeutic agents being tested in specific tumor types, but the website rarely provides much information on the biomarkers used, including imaging and genomics; simple search fields such as these could be added. We also need to speed the development of new tracers and techniques for immunotherapy imaging.

\section{CONCLUSION}

The exciting breakthroughs seen in the arena of immunotherapy require new imaging approaches to assist in the prediction and assessment of treatment response. Sophisticated analysis of relatively standard CT, MR, and PET acquisitions using radiomics approaches, as well as creation of new imaging agents, requires the efforts of many investigators across the world in the development, use, and validation of new methods.

\section{DISCLOSURE}

This work was supported by the NCI and the SNMMI. Ron H. Germain is supported by the Intramural Research Program of the National Institute of Allergy and Infectious Diseases, National Institutes of Health. No other potential conflict of interest relevant to this article was reported.

\section{REFERENCES}

1. CIP holds workshop on immune modulation therapy and imaging: immune modulation therapy and imaging - what can we do in clinical trials now? National Institutes of Health website. https://imaging.cancer.gov/news_events/ news_announcements/2016/20160705_CIP_workshop.htm. Published July 5, 2016. Updated October 28, 2016. Accessed November 29, 2017.

2. SNMMI clinical trials network co-sponsors workshop with NCI. Society of Nuclear Medicine and Molecular Imaging website. http://wwwsnmmiorg/ NewsPublications/NewsDetailaspx?ItemNumber $=15883$ ). Accessed November $29,2017$.

3. Hodi FS, O'Day SJ, McDermott DF, et al. Improved survival with ipilimumab in patients with metastatic melanoma. N Engl J Med. 2010;363:711-723.

4. Larkin J, Hodi FS, Wolchok JD. Combined nivolumab and ipilimumab or monotherapy in untreated melanoma. N Engl J Med. 2015;373:1270-1271.

5. Spranger S, Luke JJ, Bao R, et al. Density of immunogenic antigens does not explain the presence or absence of the T-cell-inflamed tumor microenvironment in melanoma. Proc Natl Acad Sci USA. 2016;113:E7759-E7768.

6. Gros A, Robbins PF, Yao X, et al. PD-1 identifies the patient-specific CD8(+) tumor-reactive repertoire infiltrating human tumors. J Clin Invest. 2014;124:22462259.

7. Keir ME, Butte MJ, Freeman GJ, Sharpe AH. PD-1 and its ligands in tolerance and immunity. Annu Rev Immunol. 2008;26:677-704.

8. Balar AV, Weber JS. PD-1 and PD-L1 antibodies in cancer: current status and future directions. Cancer Immunol Immunother. 2017;66:551-564.

9. Lum LG, Thakur A, Al-Kadhimi Z, et al. Targeted T-cell therapy in stage IV breast cancer: a phase I clinical trial. Clin Cancer Res. 2015;21:23052314 .

10. Grabert RC, Cousens LP, Smith JA, et al. Human T cells armed with Her2/neu bispecific antibodies divide, are cytotoxic, and secrete cytokines with repeated stimulation. Clin Cancer Res. 2006;12:569-576.

11. Seymour L, Bogaerts J, Perrone A, et al. iRECIST: guidelines for response criteria for use in trials testing immunotherapeutics. Lancet Oncol. 2017;18: e143-e152.

12. Reusch U, Sundaram M, Davol PA, et al. Anti-CD3 $\times$ anti-epidermal growth factor receptor (EGFR) bispecific antibody redirects T-cell cytolytic activity to EGFR-positive cancers in vitro and in an animal model. Clin Cancer Res. 2006;12:183-190.

13. Soares KC, Rucki AA, Wu AA, et al. PD-1/PD-L1 blockade together with vaccine therapy facilitates effector T-cell infiltration into pancreatic tumors. $J$ Immunother. 2015;38:1-11.

14. Le DT, Lutz E, Uram JN, et al. Evaluation of ipilimumab in combination with allogeneic pancreatic tumor cells transfected with a GM-CSF gene in previously treated pancreatic cancer. J Immunother. 2013;36:382-389.

15. Sharma P, Allison JP. Immune checkpoint targeting in cancer therapy: toward combination strategies with curative potential. Cell. 2015;161:205-214.

16. Kirkwood JM, Butterfield LH, Tarhini AA, Zarour H, Kalinski P, Ferrone S. Immunotherapy of cancer in 2012. CA Cancer J Clin. 2012;62:309-335.

17. George S, Motzer RJ, Hammers HJ, et al. Safety and efficacy of nivolumab in patients with metastatic renal cell carcinoma treated beyond progression: a subgroup analysis of a randomized clinical trial. JAMA Oncol. 2016;2:1179-1186.

18. Wolchok JD, Hoos A, O'Day S, et al. Guidelines for the evaluation of immune therapy activity in solid tumors: immune-related response criteria. Clin Cancer Res. 2009;15:7412-7420.

19. Mozley PD, Bendtsen C, Zhao B, et al. Measurement of tumor volumes improves RECIST-based response assessments in advanced lung cancer. Transl Oncol. 2012;5:19-25.

20. Suryawanshi S, Godfrey CJ, Roy A, Simonsen K, French J, Gupta M. Leveraging longitudinal tumor data for prediction of overall survival from I-O therapy: a proof of concept with quantitative models and external validation [abstract]. $J$ Pharmacokinet Pharmacodyn. 2015;42(suppl 1):M-057.

21. Graham MM, Weber WA. Evaluation of the efficacy of targeted imaging agents. J Nucl Med. 2016;57:653-659.

22. Ruella $\mathrm{M}$, June $\mathrm{CH}$. Chimeric antigen receptor $\mathrm{T}$ cells for $\mathrm{B}$ cell neoplasms: choose the right CAR for you. Curr Hematol Malig Rep. 2016;11:368-384. 
23. Topalian SL, Drake CG, Pardoll DM. Immune checkpoint blockade: a common denominator approach to cancer therapy. Cancer Cell. 2015;27:450-461.

24. Iida N, Dzutsev A, Stewart CA, et al. Commensal bacteria control cancer response to therapy by modulating the tumor microenvironment. Science. 2013;342:967-970.

25. Gerner MY, Kastenmuller W, Ifrim I, Kabat J, Germain RN. Histo-cytometry: a method for highly multiplex quantitative tissue imaging analysis applied to dendritic cell subset microanatomy in lymph nodes. Immunity. 2012;37:364-376.

26. Chen DS, Mellman I. Oncology meets immunology: the cancer-immunity cycle. Immunity. 2013;39:1-10.

27. Kwak JJ, Tirumani SH, Van den Abbeele AD, Koo PJ, Jacene HA. Cancer immunotherapy: imaging assessment of novel treatment response patterns and immune-related adverse events. Radiographics. 2015;35:424-437.

28. Eisenhauer EA, Therasse P, Bogaerts J, et al. New response evaluation criteria in solid tumours: revised RECIST guideline (version 1.1). Eur J Cancer. 2009;45: 228-247.

29. Young H, Baum R, Cremerius U, et al. Measurement of clinical and subclinical tumour response using $\left[{ }^{18} \mathrm{~F}\right]$-fluorodeoxyglucose and positron emission tomography: review and 1999 EORTC recommendations. European Organization for Research and Treatment of Cancer (EORTC) PET Study Group. Eur J Cancer. 1999;35:1773-1782.

30. Tumeh PC, Harview CL, Yearley JH, et al. PD-1 blockade induces responses by inhibiting adaptive immune resistance. Nature. 2014;515:568-571.

31. Ribas A, Benz MR, Allen-Auerbach MS, et al. Imaging of CTLA4 blockadeinduced cell replication with ${ }^{18} \mathrm{~F}$-FLT PET in patients with advanced melanoma treated with tremelimumab. J Nucl Med. 2010;51:340-346.

32. Juergens RA, Zukotynski KA, Singnurkar A, Snider DP, Valliant JF, Gulenchyn KY. Imaging biomarkers in immunotherapy. Biomark Cancer. 2016;8(suppl 2):1-13.

33. Kumar V, Gu Y, Basu S, et al. Radiomics: the process and the challenges. Magn Reson Imaging. 2012;30:1234-1248.

34. Lamberts LE, Williams SP, Terwisscha van Scheltinga AG, et al. Antibody positron emission tomography imaging in anticancer drug development. J Clin Oncol. 2015;33:1491-1504.

35. Jauw YW, Menke-van der Houven van Oordt CW, Hoekstra OS, et al. Immunopositron emission tomography with zirconium-89-labeled monoclonal antibodies in oncology: what can we learn from initial clinical trials? Front Pharmacol. 2016;7:131.

36. Goldenberg DM. Perspectives on oncologic imaging with radiolabeled antibodies. Cancer. 1997;80(12, suppl):2431-2435.

37. Gebhart G, Lamberts LE, Wimana Z, et al. Molecular imaging as a tool to investigate heterogeneity of advanced HER2-positive breast cancer and to predict patient outcome under trastuzumab emtansine (T-DM1): the ZEPHIR trial. Ann Oncol. 2016;27:619-624.
38. Heskamp S, Hobo W, Molkenboer-Kuenen JD, et al. Noninvasive imaging of tumor PD-L1 expression using radiolabeled anti-PD-L1 antibodies. Cancer Res. 2015;75:2928-2936.

39. Chatterjee S, Lesniak WG, Gabrielson M, et al. A humanized antibody for imaging immune checkpoint ligand PD-L1 expression in tumors. Oncotarget. 2016;7:10215-10227.

40. Warnders FJ, Waaijer SJ, Pool M, et al. Biodistribution and PET imaging of labeled bispecific T cell-engaging antibody targeting EpCAM. J Nucl Med. 2016;57:812-817.

41. Koch M, de Jong JS, Glatz J, et al. Threshold analysis and biodistribution of fluorescently labeled bevacizumab in human breast cancer. Cancer Res. 2017; 77:623-631.

42. McCracken MN, Tavaré R, Witte ON, Wu AM. Advances in PET detection of the antitumor T cell response. Adv Immunol. 2016;131:187-231.

43. Wu AM. Engineered antibodies for molecular imaging of cancer. Methods. 2014;65:139-147.

44. Tavaré R, McCracken MN, Zettlitz KA, et al. Immuno-PET of murine T cell reconstitution postadoptive stem cell transplantation using anti-CD4 and antiCD8 cys-diabodies. J Nucl Med. 2015;56:1258-1264.

45. Tavaré R, Escuin-Ordinas H, Mok S, et al. An effective immuno-PET imaging method to monitor CD8-dependent responses to immunotherapy. Cancer Res. 2016;76:73-82.

46. Pandit-Taskar N, O'Donoghue JA, Ruan S, et al. First-in-human imaging with ${ }^{89} \mathrm{Zr}$-Df-IAB2M anti-PSMA minibody in patients with metastatic prostate cancer: pharmacokinetics, biodistribution, dosimetry, and lesion uptake. J Nucl Med. 2016;57:1858-1864.

47. Platten M, von Knebel Doeberitz N, Oezen I, Wick W, Ochs K. Cancer immunotherapy by targeting IDO1/TDO and their downstream effectors. Front Immunol. 2015;5:673.

48. Huang X, Gillies RJ, Tian H. Synthesis of $\left[{ }^{18} \mathrm{~F}\right] 4$-amino-N-(3-chloro-4fluorophenyl)-N'-hydroxy-1,2,5-oxadiazole-3-carboximidamide (IDO5L): a novel potential PET probe for imaging of IDO1 expression. J Labelled Comp Radiopharm. 2015;58:156-162.

49. Namavari M, Chang YF, Kusler B, Yaghoubi S, Mitchell BS, Gambhir SS. Synthesis of $2^{\prime}$-deoxy-2'-[ $\left.{ }^{18} \mathrm{~F}\right]$ fluoro-9-beta-D-arabinofuranosylguanine: a novel agent for imaging T-cell activation with PET. Mol Imaging Biol. 2011;13:812-818.

50. Chatterjee S, Lesniak WG, Miller MS, et al. Rapid PD-L1 detection in tumors with PET using a highly specific peptide. Biochem Biophys Res Commun. 2017;483:258-263.

51. Herbst RS, Soria JC, Kowanetz M, et al. Predictive correlates of response to the anti-PD-L1 antibody MPDL3280A in cancer patients. Nature. 2014;515:563-567. 